\title{
PREFACE TO THE 1989 EDITION
}

I'm sad to say that, having reread the preface I wrote in 1978 , I see no reason to change its overall thrust. However, my subsequent experience with the United States Department of Agriculture, in connection with the Medfly disaster, does lead me to believe that I perhaps held too high an opinion of it in 1978 .

Paul Ehrlich 
